Check for updates

Cite this: Chem. Commun., 2021, 57,11382

Received 12th August 2021

Accepted 22nd September 2021

DOI: $10.1039 / \mathrm{d} 1 \mathrm{cc} 04459 a$

rsc.li/chemcomm

\section{Design, synthesis, and initial immunological evaluation of glycoconjugates based on saponin adjuvants and the $T n$ antigen $\dagger$}

\author{
Roberto Fuentes, (D) ${ }^{a}$ Leire Aguinagalde, (D) ${ }^{a}$ Nagore Sacristán ${ }^{a}$ and \\ Alberto Fernández-Tejada (iD *ab
}

\begin{abstract}
We report the first synthesis and immunological evaluation of a new glycoconjugate design based on streamlined saponin adjuvants and the Tn carbohydrate antigen. While the novel synthetic constructs induced moderate antibody responses in mice, the versatile chemical platform is amenable to further structure-activity optimizations for the development of self-adjuvanting glycoconjugate cancer vaccines.
\end{abstract}

The Tn antigen ( $N$-acetylgalactosamine (GalNAc) $\alpha$-O-linked to serine/threonine) is a typical tumour associated carbohydrate antigen (TACA) overexpressed on cell surface glycoproteins in human tumours. Despite its potential for anti-cancer vaccine development, it is weakly immunogenic and needs to be administered as a conjugate to induce robust immune responses. ${ }^{1}$ The classical approach involves covalent attachment to an immunogenic carrier protein leading to enhanced antigen presentation and $\mathrm{T}$ cell activation, ${ }^{2}$ and coadministration with an immunological adjuvant such as the saponin natural product QS-21, which activates both antibody and cellular immunity, ${ }^{3}$ further potentiating the immune response. QS-21 is a mixture of triterpenoid glycoside isomers (QS-21-api, 1a and QS-21-xyl, 1b) that share a central quillaic acid (QA) triterpene flanked by a left-hand branched trisaccharide and a C28-linked linear tetrasaccharide decorated with a glycosylated acyl chain (Fig. 1a). Despite its wide clinical application and recent approvals as part of the AS01 adjuvant system, ${ }^{4}$ the scarcity, heterogeneity, chemical instability and dose-limiting toxicity of the natural product have impeded the advancement of QS-21 as a stand-alone adjuvant in vaccines. ${ }^{5}$ Notably, a range of synthetic derivatives developed by Gin, Fernández-Tejada and coworkers ${ }^{6-9}$ have provided streamlined chemical access to simplified, amide-stabilized QS-21 variants, most notably lacking the left-hand carbohydrate domain and the

\footnotetext{
${ }^{a}$ Chemical Immunology Laboratory, CIC bioGUNE, Basque Research and Technology Alliance (BRTA), 48160 Derio, Spain.

E-mail: afernandeztejada@cicbiogune.es

${ }^{b}$ Ikerbasque, Basque Foundation for Science, Euskadi Plaza 5, 48009 Bilbao, Spain $\dagger$ Electronic supplementary information (ESI) available. See DOI: 10.1039/ d1cc04459a
}

apiose/xylose residue. In preclinical settings, some of these sugartruncated saponin analogues, specifically those derived from QA and the related echinocystic acid (EA) triterpene (which differs at its C4-methyl group), showed negligible toxicity and potent adjuvant activity, with induction of antibody responses close to those observed with QS-21. ${ }^{10,11}$ These promising immunological features, together with their improved synthetic accessibility, highlight their potential for further development in novel subunit vaccines, a prospect under-investigated so far.

An alternative concept that overcomes the issues of heterogeneity and the carrier-induced epitope suppression of the protein conjugate vaccines is the so-called "self-adjuvanting", approach. This relies on synthetic constructs incorporating well

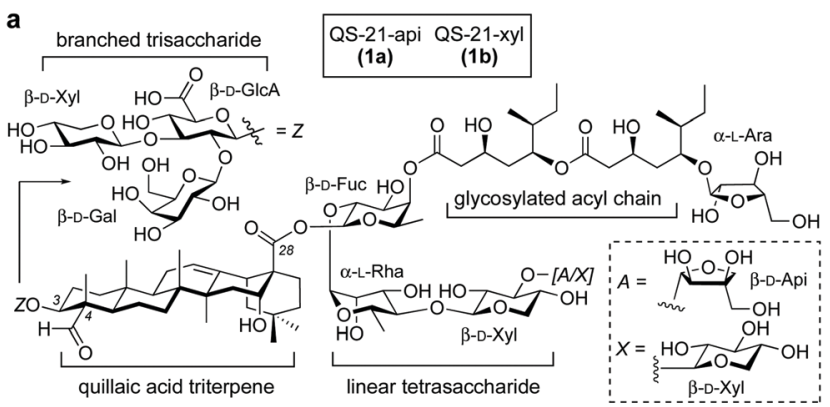

b

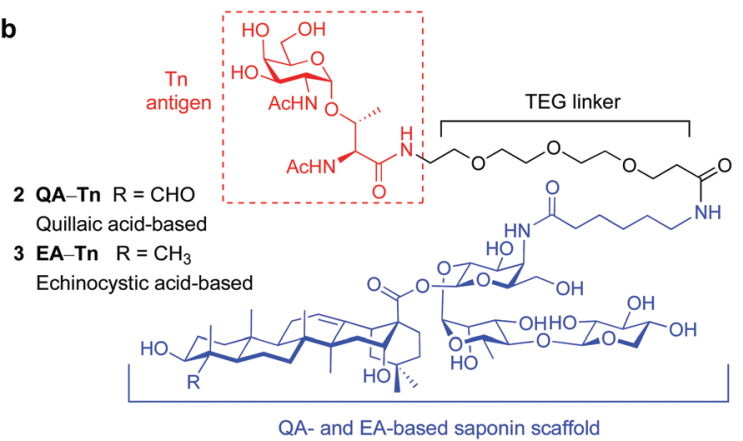

Fig. 1 (a) Chemical structure of QS-21 natural product adjuvant (1a/1b), and (b) streamlined saponin-Tn conjugates (2 QA-Tn and 3 EA-Tn). 
defined antigen and adjuvants components in the same molecule, aiming at achieving enhanced TACA presentation and recognition by the immune system leading to a more focused antigen-specific response. ${ }^{12}$ Several types of such constructions feature the Tn antigen (or related mucin-type TACAs) covalently linked to diverse built-in adjuvant moieties, mostly derived from TLR ligands (e.g. Pam ${ }_{3}$ Cys derivatives, monophosphoryl lipid A (MPLA)) and the invariant natural killer T (iNKT) cell ligand $\alpha$-galactosylceramide ( $\alpha$-GalCer). ${ }^{13}$ In early examples, ${ }^{14,15}$ $\mathrm{Tn}$ (Ser) units were attached to the TLR2 agonist Pam ${ }_{3}$ CysSer immunopotentiator, providing synthetic conjugates that induced modest Tn-specific antibody responses in mice. Other di- and tri-component Tn-based immunogens have also been developed incorporating TLR2 ligands and T cell epitopes, inducing strong and functional anti-Tn IgG antibodies in mice even in the absence of external adjuvants. ${ }^{16-18}$ More recently, Tn and other carbohydrate antigens have been chemically linked to MPLA $^{19-21}$ and $\alpha$-GalCer ${ }^{22,23}$ glycolipids, yielding novel types of dicomponent glycoconjugates that induced Tn-specific IgG antibodies when administered in liposomes. Besides the corresponding mucinlike $O$-glycopeptide conjugates synthesized and evaluated by many laboratories, ${ }^{24-27}$ additional Tn-presenting scaffolds have been investigated as vaccine candidates, including cyclopeptides, ${ }^{28}$ virus-like particles $^{29}$ and polysaccharide-based carriers (e.g. PS A-1). ${ }^{30}$

However, despite its promise, ${ }^{31}$ saponin-derived adjuvants have not been exploited so far for chemical conjugation to synthetic antigens, making the development of such saponinbased molecular vaccines unprecedented. Thus, we sought to leverage the promising properties of our minimal synthetic saponin analogues to apply them, for the first time, in the construction of novel, minimalistic adjuvant-antigen glycoconjugates as potential "self-adjuvanting" vaccine candidates. Adjuvant-active saponin variants have been previously demonstrated to be internalized by dendritic cells, ${ }^{32}$ which play a role in adjuvant activity and are involved in the uptake, processing and presentation of antigens to $\mathrm{T}$ cells, leading to activation of adaptive immunity. Therefore, we hypothesized that, by colocalizing both components in the same molecule through covalent linkage of the Tn antigen to the saponin scaffold, this adjuvant platform could assist in delivering the conjugate construct (and consequently the attached antigen) to the same antigen presenting cell, thus enhancing its uptake and presentation to the adaptive immune system and potentially increasing the immune response. In developing this fully synthetic approach, streamlined synthesis, structural simplicity and facile conjugation chemistry were our key design criteria to facilitate accessibility, full chemical characterization and easy manipulation of the resulting glycoconjugate construct. Herein, we report the first chemical synthesis and initial immunological evaluation of a novel carbohydrate-based vaccine design consisting of two minimal saponin-Tn conjugates ( 2 and 3 ) (Fig. 1b), which leverage optimal triterpene cores and a wellestablished site for modification (i.e. acyl chain terminus) with retention of saponin adjuvant activity. Preliminary in vivo immunological studies suggest that this first dicomponent design induces a moderate antibody response against the weakly immunogenic carbohydrate antigen Tn. Notably, the versatility of the chemical strategy as well as the presence of key sites for chemical modification in the saponin scaffold provides additional opportunities for further structure-activity optimization currently underway in our laboratories.

In terms of chemical design of the candidates, our previous development of unimolecular adjuvant conjugates by covalently linking the immunopotentiator tucaresol to saponin adjuvants at the side chain terminal amine ${ }^{6}$ indicated that these saponins are amenable to significant structural modification at the acyl chain terminus without impairing adjuvant activity. Thus, we decided to exploit this privileged position in our minimal saponin scaffolds for covalent attachment of well-defined carbohydrate antigens to generate novel glycoconjugate vaccine constructs. To test our hypothesis and probe the selfadjuvanting potential of our saponin platform, we selected the Tn antigen as the prototypical TACA for the synthetic construction of minimalistic saponin-Tn glycoconjugates 2 and 3. As saponin scaffolds, we chose 6-aminohexanoic amide variants 9 and $\mathbf{1 0}^{\mathbf{1 0}}$ (Scheme 1), which are derived from our sugar-truncated lead compounds containing the quillaic acid (QA) and echinocystic acid (EA) triterpenes, respectively. ${ }^{11}$ In place of the longer carboxylic acid terminating side chain present in the lead saponins, analogues $\mathbf{9}$ and $\mathbf{1 0}$ incorporate a six-carbon acyl chain with a primary amine at its end that enables chemoselective functionalization of the fully deprotected molecule with conveniently activated counterparts. Thus, we envisioned that the Tn antigen could be attached to the terminal amino group of the saponins by amidation of a properly derivatized $\mathrm{Tn}(\mathrm{Thr})$ glycoamino acid building block (8) (Scheme 1).

This compound was equipped with a triethyleneglycol (TEG) spacer to improve water solubility of the resulting conjugate and was readily synthesized in three steps starting from a previously reported precursor $(4)^{17}$ (Scheme 1a). Briefly, Fmocprotected $\mathrm{Ac}_{3}$-GalNAc- $\alpha-\mathrm{O}-\mathrm{Thr}$ acid $\mathbf{4}$ was first elongated with

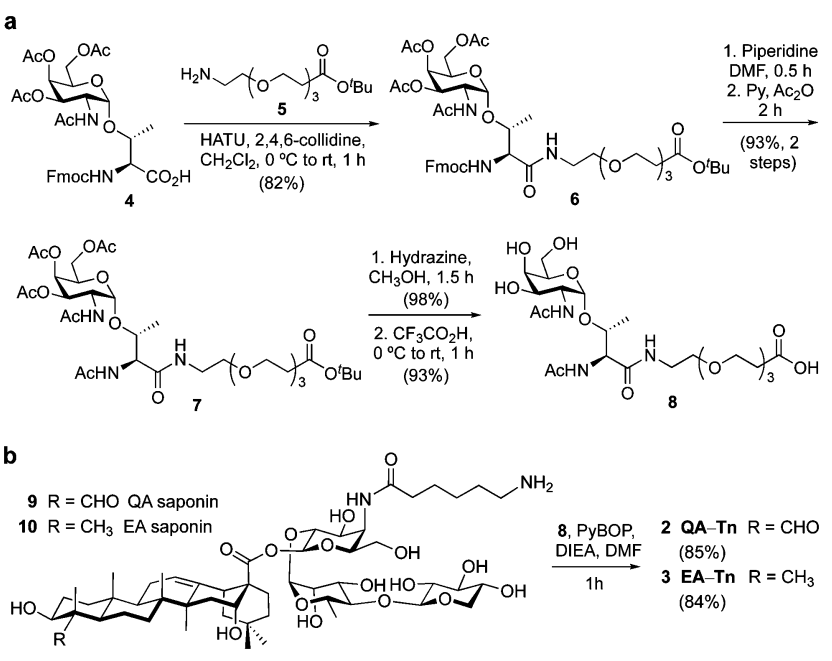

Scheme 1 Synthesis of (a) spacer-containing Tn antigen 8 and (b) saponin-Tn conjugates 2 ( $Q A-T n$ ) and 3 (EA-Tn). 
amino- $\mathrm{PEG}_{3}-t$-butyl ester (5) via amidation under carefully controlled conditions to avoid racemization (HATU, collidine, $\mathrm{CH}_{2} \mathrm{Cl}_{2}, 0{ }^{\circ} \mathrm{C}, 1 \mathrm{~h}$ ), providing spacer-containing $\mathrm{Tn}$ intermediate 6 in $82 \%$ yield. Fmoc removal (piperidine, DMF) and subsequent acetylation (pyridine, $\mathrm{Ac}_{2} \mathrm{O}$ ) of the threonine amino group afforded compound 7 in 93\% yield over two steps. Finally, deprotection of the sugar hydroxyl groups (hydrazine, $\mathrm{MeOH}$ ) followed by removal of the linker $t$-butyl ester (TFA, $0{ }^{\circ} \mathrm{C}, 1 \mathrm{~h}$ ) yielded the derivatized $\mathrm{Tn}(\mathrm{Thr})$ antigen 8 (93\% yield, two steps) ready for conjugation through the free carboxylic acid of the TEG chain. The key coupling step between this Tn moiety (8) and the amine-terminating saponin scaffold $(\mathbf{9}, \mathbf{1 0})$ involved straightforward amide formation using PyBOP as in situ activator in the presence of DIEA as a base, providing the desired QA and EA-derived saponin-Tn conjugates (2 QA-Tn and $3 \mathrm{EA}-\mathrm{Tn}$ ) in $85 \%$ and $84 \%$ yield, respectively (Scheme $1 \mathrm{~b}$ ). For use as a coating antigen in the ELISA analysis, the corresponding Tn moiety was conjugated to bovine serum albumin (BSA-Tn) via initial activation of the acid 8 as NHS ester (DCC/NHS, DMF), followed by coupling to the protein in phosphate buffer saline (PBS). The resulting conjugate was determined to have an average number of 9 copies of Tn moiety per BSA, as measured by MALDI (see $\mathrm{ESI} \dagger$ for details).

We next assessed the immunogenicity of the synthetic constructs for their ability to elicit Tn-specific antibody responses in an initial in vivo evaluation. Groups of five mice (female C57BL/6) were immunized subcutaneously (100 $\mu \mathrm{l}$ injections) three times every two weeks (days 0, 14, and 28) with both glycoconjugates (60 $\mu \mathrm{g}$ dose per mouse) dissolved in PBS. As a negative control, a mock group was injected with PBS alone. Mice were bled one week before (day 21) and two weeks after the third immunization at the time of sacrifice (day 42) and the presence of anti-Tn antibodies at days 21 and 42 was determined by ELISA. In addition to IgM antibodies, mice immunized with QA-Tn (2) and EA-Tn (3) showed comparable antibody responses on day 42 in terms of IgG levels, which albeit modest, were increased compared to those induced on day 21 and significantly higher than the levels observed in the mock group (Fig. 2). To assess further the type of immune response elicited by the constructs, subtyping of the IgG antibodies at day 42 was performed. This analysis revealed induction of generally increased IgG2b and IgG2c levels (which in the case of the group administered 2 were significantly higher than those observed with the negative control) and to a lesser extent IgG3, whereas, in comparison, lower levels of IgG1 antibodies relative to the control group were observed (Fig. S3, ESI $\dagger$ ). As the mouse IgG1 subtype is generally associated to Th2 responses and Th1 immunity primarily favours production of IgG2 (and IgG3) subclass, our results could suggest a bias towards a Th1-type IgG response for both saponin-Tn conjugates. Notably, based on the lack of mouse weight loss as assessed at 0, 24, 48 and $72 \mathrm{~h}$ post-injection (Fig. 3) and by visual inspection, no toxic side effects (local inflammation, systemic reactions or death) were observed over the course of the immunization, highlighting the biosafety of both conjugates.
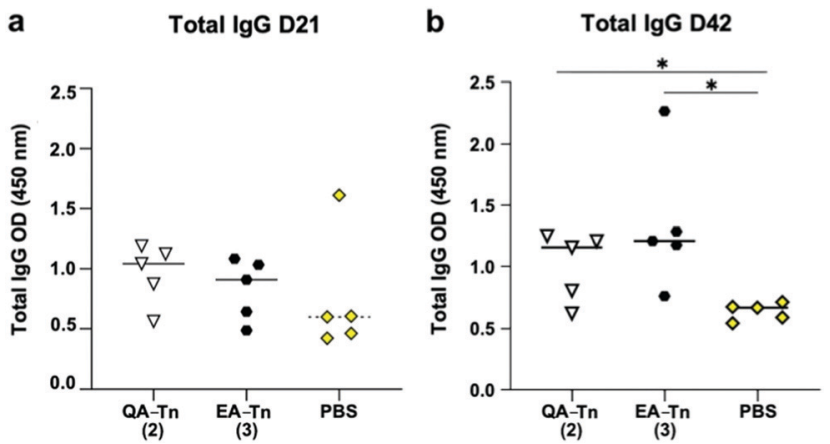

Fig. 2 Antibody responses in mice induced by saponin-Tn conjugates 2 and 3. Total anti-Tn IgG levels in mouse sera on (a) day 21 and (b) day 42 after first immunization detected by ELISA using a 1/50 serum dilution. Horizontal bars indicate optical density (OD) median values. Statistical significance versus negative control group (PBS only) was determined using two-sided unpaired Student's $t$-test with ${ }^{*} p \leq 0.05$.

In conclusion, we have developed two new well-defined, fully synthetic adjuvant-antigen glycoconjugates in a first example of dicomponent vaccine constructs involving saponin variants chemically linked to TACAs. The preliminary immunological evaluation in mice revealed that this novel saponin-Tn conjugate design could induce a certain degree of Tn-specific antibodies in the absence of any external adjuvants or carrier systems. While this "self-adjuvanting" approach is expected to benefit from additional structural and/or formulation optimization, the streamlined synthetic strategy towards both saponin and Tn components as well as the versatile chemical design and facile conjugation via straightforward amide linkage opens the door to further improve upon this initial promising platform. Moreover, the exploration of special pharmaceutical preparations of the constructs could also contribute to increase their immunogenicity. Efforts in these directions are currently ongoing in our laboratory in search of more potent synthetic vaccine candidates based on conjugates of saponin adjuvants and the Tn antigen as well as other antigenic epitopes.

R. F. performed the chemical synthesis of the constructs. L. A. carried out the immunological experiments with the assistance of

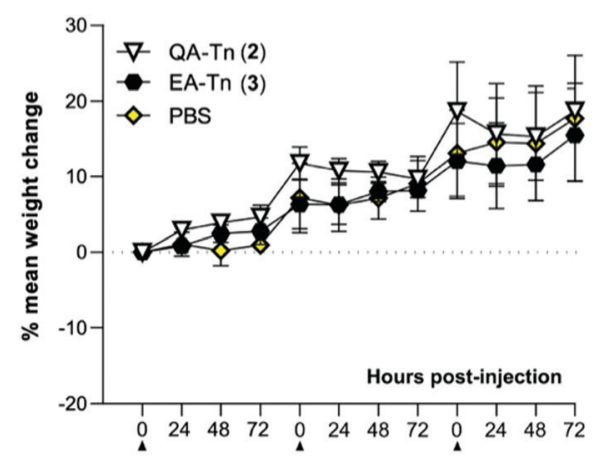

Fig. 3 Overall toxicity assessment based on mouse weight change at 0 -, 24-, 48 and 72 hours post-injection, indicating non-toxicity of the saponin-Tn conjugates. Arrows represent immunization days $(0,14$ and 28, respectively) 
N. S. and interpreted the relevant data. A. F.-T. designed and supervised the entire research, discussed the results with the team, and wrote the manuscript. All authors approved the submitted version.

This work is dedicated to the memory of Prof. David Y. Gin (1967-2011) on the 10 year memorial of his passing. Funding from the ERC (ERC-2016-STG-716878 to A. F.-T.) and the Spanish Ministry of Science and Innovation (CTQ2017-87530-R, RYC-201517888 to A. F.-T.) is acknowledged. We thank Dr Carlo Pifferi for technical assistance. A. F.-T. thanks Raquel Fernández for inspiration.

\section{Conflicts of interest}

There are no conflicts to declare.

\section{Notes and references}

1 T. Buskas, P. Thompson and G. J. Boons, Chem. Commun., 2009, 5335-5349.

2 R. M. Wilson and S. J. Danishefsky, J. Am. Chem. Soc., 2013, 135, 14462-14472.

3 G. Ragupathi, J. R. Gardner, P. O. Livingston and D. Y. Gin, Expert Rev. Vaccines, 2011, 10, 463-470.

4 M. A. Lacaille-Dubois, Phytomedicine, 2019, 60, 152905.

5 C. Pifferi, R. Fuentes and A. Fernández-Tejada, Nat. Rev. Chem., 2021, 5, 197-216.

6 A. Fernández-Tejada, E. K. Chea, C. George, J. R. Gardner, P. O. Livingston, G. Ragupathi, D. S. Tan and D. Y. Gin, Bioorg. Med. Chem., 2014, 22, 5917-5923.

7 A. Fernández-Tejada, D. S. Tan and D. Y. Gin, Chem. Commun., 2015, 51, 13949-13952.

8 A. Fernández-Tejada, Pure Appl. Chem., 2017, 89, 1359-1378.

9 R. Fuentes, A. Ruiz-de-Angulo, N. Sacristán, C. D. Navo, G. JiménezOsés, J. Anguita and A. Fernández-Tejada, Chem. - Eur. J., 2021, 27, 4731-4737.

10 A. Fernández-Tejada, E. K. Chea, C. George, N. Pillarsetty, J. R. Gardner, P. O. Livingston, G. Ragupathi, J. S. Lewis, D. S. Tan and D. Y. Gin, Nat. Chem., 2014, 6, 635-643.

11 M. Ghirardello, A. Ruiz-De-Angulo, N. Sacristan, D. Barriales, J. Jiménez-Barbero, A. Poveda, F. Corzana, J. Anguita and A. Fernández-Tejada, Chem. Commun., 2020, 56, 719-722.

12 W. H. Li and Y. M. Li, Chem. Rev., 2020, 120, 11420-11478.
13 S. Lang and X. Huang, Front. Chem., 2020, 8, 1-25.

14 T. Toyokuni, B. Dean, S. Cai, D. Boivin, S. Hakomori and A. K. Singhal, J. Am. Chem. Soc., 1994, 116, 395-396.

15 S. D. Kuduk, J. B. Schwarz, X. T. Chen, P. W. Glunz, D. Sames, G. Ragupathi, P. O. Livingston and S. J. Danishefsky, J. Am. Chem. Soc., 1998, 120, 12474-12485.

16 R. Lo-Man, S. Vichier-Guerre, S. Bay, E. Dériaud, D. Cantacuzène and C. Leclerc, J. Immunol., 2001, 166, 2849-2854.

17 T. Buskas, S. Ingale and G. J. Boons, Angew. Chem., Int. Ed., 2005, 44, $5985-5988$

18 A. B. M. Abdel-Aal, D. El-Naggar, M. Zaman, M. Batzloff and I. Toth, J. Med. Chem., 2012, 55, 6968-6974.

19 Q. Wang, Z. Zhou, S. Tang and Z. Guo, ACS Chem. Biol., 2012, 7, 235-240.

20 Z. Zhou, M. Mondal, G. Liao and Z. Guo, Org. Biomol. Chem., 2014, 12, 3238-3245.

21 Z. Zhou, G. Liao, S. S. Mandal, S. Suryawanshi and Z. Guo, Chem. Sci., 2015, 6, 7112-7121.

22 X. G. Yin, X. Z. Chen, W. M. Sun, X. S. Geng, X. K. Zhang, J. Wang, P. P. Ji, Z. Y. Zhou, D. J. Baek, G. F. Yang, Z. Liu and J. Guo, Org. Lett., 2017, 19, 456-459.

23 F. Broecker, S. Götze, J. Hudon, D. C. K. Rathwell, C. L. Pereira, P. Stallforth, C. Anish and P. H. Seeberger, J. Med. Chem., 2018, 61, 4918-4927.

24 D. M. McDonald, S. N. Byrne and R. J. Payne, Front. Chem., 2015, 3, 1-8.

25 S. Ingale, M. A. Wolfert, J. Gaekwad, T. Buskas and G. J. Boons, Nat. Chem. Biol., 2007, 3, 663-667.

26 V. Lakshminarayanan, P. Thompson, M. A. Wolfert, T. Buskas, J. M. Bradley, L. B. Pathangey, C. S. Madsen, P. A. Cohen, S. J. Gendler and G.-J. Boons, Proc. Natl. Acad. Sci. U. S. A., 2012, 109, 261-266.

27 N. Gaidzik, U. Westerlind and H. Kunz, Chem. Soc. Rev., 2013, 42, 4421-4442.

28 C. Pifferi, A. Ruiz-De-Angulo, D. Goyard, C. Tiertant, N. Sacristán, D. Barriales, N. Berthet, J. Anguita, O. Renaudet and A. FernándezTejada, Chem. Sci., 2020, 11, 4488-4498.

29 Z. Yin, S. Chowdhury, C. McKay, C. Baniel, W. S. Wright, P. Bentley, K. Kaczanowska, J. C. Gildersleeve, M. G. Finn, L. BenMohamed and X. Huang, ACS Chem. Biol., 2015, 10, 2364-2372.

30 R. A. De Silva, Q. Wang, T. Chidley, D. K. Appulage and P. R. Andreana, J. Am. Chem. Soc., 2009, 131, 9622-9623.

31 A. Fernández-Tejada, D. S. Tan and D. Y. Gin, Acc. Chem. Res., 2016, 49, 1741-1756.

32 E. K. Chea, A. Fernández-Tejada, P. Damani, M. M. Adams, J. R. Gardner, P. O. Livingston, G. Ragupathi and D. Y. Gin, J. Am. Chem. Soc., 2012, 134, 13448-13457. 